\title{
X-RAY DIFFRACTION MEASUREMENTS FOR EXPANDED LIQUID MERCURY AROUND THE METAL- NONMETAL TRANSITION REGION
}

\author{
K. TAMURA and S. HOSOKAWA \\ Faculty of Integrated Arts and Sciences, Hiroshima University, Hiroshima 730, Japan
}

\begin{abstract}
The energy-dispersive X-ray diffraction measurements for expanded liquid $\mathrm{Hg}$ have been carried out for the first time in the density region from 13.55 to $8.78 \mathrm{~g} / \mathrm{cm}^{3}$ including the metal-nonmetal transition region. It was found that the volume expansion of liquid $\mathrm{Hg}$ in the metallic region, namely down to the reduced density of about 10 $\mathrm{g} / \mathrm{cm}^{3}$, is not accompanied by a uniform increase of interatomic distance, $\mathrm{r} 1$, but mainly caused by a decrease of coordination number, $N_{1}$. When the transition region is approached the decrease of $\mathrm{N}_{1}$ becomes rather small and $\mathrm{r}_{1}$ starts to elongate.
\end{abstract}

\section{Introduction}

A great deal of efforts have been devoted to the study on the electronic and thermodynamic properties of fluid $\mathrm{Hg}$ over the range of temperature and pressure sufficiently wide to include the liquid-gas critical point (critical data of $\mathrm{Hg} / 1 /: \mathrm{T}_{\mathrm{c}}=1478^{\circ} \mathrm{C}, \mathrm{p}_{\mathrm{c}}=1673 \mathrm{bar}, \mathrm{d}_{\mathrm{c}}=5.8 \mathrm{~g} / \mathrm{cm}^{3}$ ). Much of the efforts have been concerned with the metal-nonmetal (M-NM) transition which occurs in the liquid when it is expanded beyond the critical point. A number of measurements of physical properties such as electrical conductivity /2-5/, thermopower /3,6-8/, Hall coefficient /9/, optical reflectivity $/ 10,11 /, \mathrm{NMR} / 12 /$, sound velocity $/ 13 /$ and equation of state $/ 1,2,4,5 /$ show that, as the density is reduced, the M-NM transition starts to occur at the density of about $9 \mathrm{~g} / \mathrm{cm}^{3}$.

It is obvious that the structural data around the transition region are quite important to the understandings of the M-NM transition. However, information on the structure of expanded liquid $\mathrm{Hg}$ has been quite limited mainly because there have been several technical problems accompanied by such an extreme experimental condition. Recently we have reported for the first time the structural data of expanded liquid $\mathrm{Hg}$ in the temperature and pressure range up to $1200^{\circ} \mathrm{C}$ and $830 \mathrm{bar}$, in the density range between 13.55 and $10.26 \mathrm{~g} / \mathrm{cm}^{3} / 14 /$, by the energy-dispersive $\mathrm{X}$ ray diffraction method using new type of high pressure vessel and sample cell developed in our laboratory $/ 14,15 /$. We have obtained the result that the nearest neighbour distance, $\mathrm{r}_{1}$, remains almost constant in the measured density range and the coordination number, $\mathrm{N}_{1}$, decreases substantially and almost linearly with decreasing density /14/. It is interesting to study how liquid $\mathrm{Hg}$ is expanded with further decrease of density beyond the M-NM transition region.

In this paper we report the results of the X-ray diffraction measurements for expanded liquid $\mathrm{Hg}$ in the temperature and pressure range up to $1450^{\circ} \mathrm{C}$ and 1750 bar, in the density region 
from 13.55 to $8.78 \mathrm{~g} / \mathrm{cm}^{3}$ including the M-NM transition region.

\section{Experimental}

We have carried out X-ray diffraction measurements for expanded liquid $\mathrm{Hg}$ using the energy-dispersive method. This technique has already been employed in our structural studies of expanded liquid Se near the liquid-gas critical point /15/ and expanded liquid $\mathrm{Hg}$ in the metallic region /14/. For the measurements of expanded liquid $\mathrm{Hg}$ in the lower density region, we have constructed another new internally heated autoclave. Figure 1 shows the side view of the autoclave which was constructed of a main cylinder with an smaller inner-diameter of $58 \mathrm{~mm}$ than that of the previous one and two flanges. As seen in the figure, the sample was located at the center of the autoclave. The primary and scattered X-ray beams passed through Be windows with the thickness of $5 \mathrm{~mm}$ and diameter of $4 \mathrm{~mm}$. The Be windows were supported by the flat area of a screws. Figure 2 shows the top view of the autoclave. As seen in the figure, there were eight windows, one of which was for the incident X-ray beam and the rest for the scattered ones. The windows for the scattered $X$-ray beams were located at the scattering angle, $2 \theta$, of $5,7.5,11,15$, 20,25 and $35^{\circ}$. He gas was used as pressurizing medium.

Expanded liquid $\mathrm{Hg}$ must be contained in a cell made of special material being transparent to X-ray and resistant to chemical corrosion by $\mathrm{Hg}$ at high temperature. A single-crystal sapphire cell was developed for the purpose, the construction of which is illustrated in figure 3(a). The details around the liquid sample is also shown in figure $3(\mathrm{~b})$ on an enlarged scale. The cell construction is essentially the same as the previous ones $/ 14,15 /$ but the size has become much small-

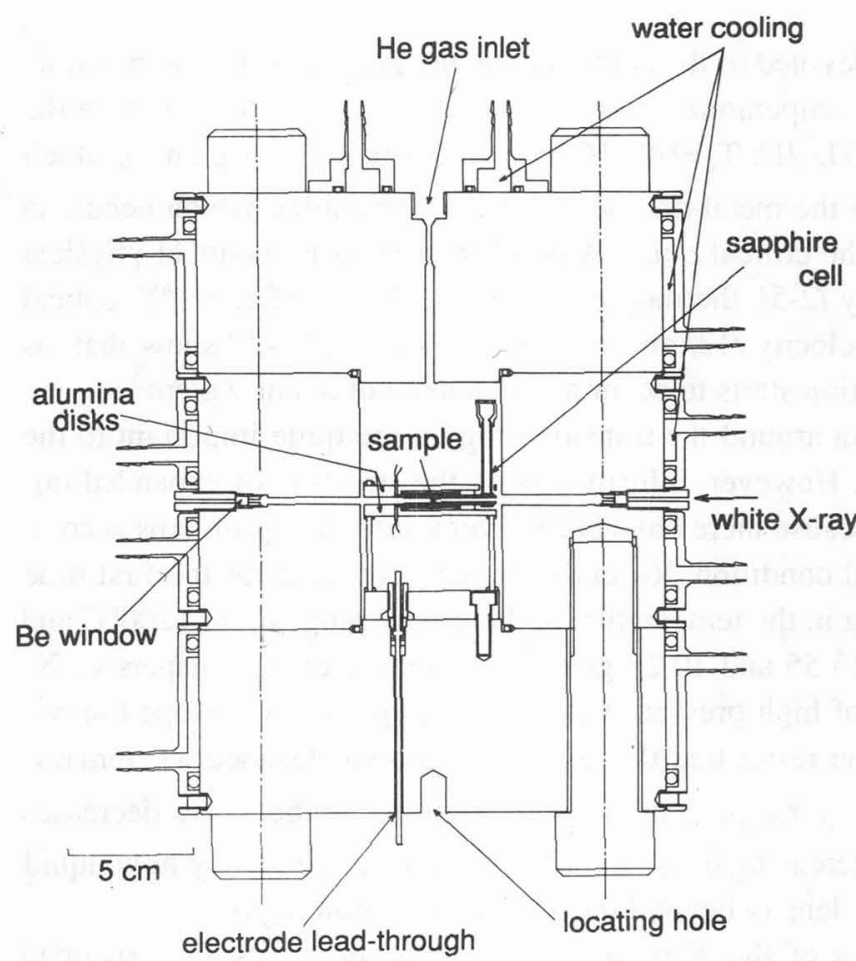

Figure 1. er. A closed-end sapphire tube with inner diameter $2.5 \mathrm{~mm}$, outer diameter $3.5 \mathrm{~mm}$ was put into another closedend tube with the inner diameter 3.5 $\mathrm{mm}$, outer diameter $4.5 \mathrm{~mm}$. The sample reservoir was connected to the outsides of the tubes. The sapphire components were cemented with a high-temperature glaze providing a uniform gap between the closed ends of the tubes as shown in figure $3(\mathrm{~b})$. The thickness of the closed ends was $150 \mu \mathrm{m}$ and that of the gap was 15 um. In measuring $X$-ray diffraction from liquid sample contained in the sapphire cell, the problem is that the strong Bragg diffraction from the sapphire cell may disturb the obtained Xray spectrum. This was overcome by imploying the suitable procedure on the cell construction $/ 14,15 /$.

The cell was heated by a heating Side view of the autoclave for X-ray diffraction studies. element made of $W$ wire with diameter 
$0.4 \mathrm{~mm}$. The temperature of the sample was measured by two Pt-30\%Rh:Pt-6\%Rh thermocouples which were located in the holes of Mo tube and were close contact with the wall of the closed end of the outer sapphire tube. It is noticed that a slit was made on the left hand side of the Mo tube so diffracted $\mathrm{X}$-rays were able to go out through it. The cell and the heater were held by alumina disks as seen in figure 1 . The space between the alumina disks and the inner wall of the autoclave was filled with alumina powder to obtain good thermal insulation and to prevent convection of the compressed He gas. As seen in figure 3(a) liquid $\mathrm{Hg}$ in the sample space was connected with that in the

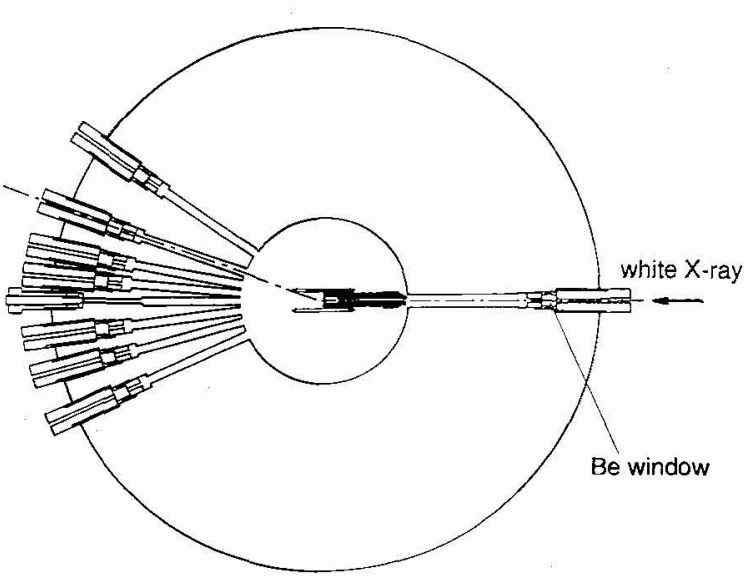

Figure 2.

Top view of the autoclave for X-ray diffraction studies sample reservoir through a small path. The balance between the compressed liquid $\mathrm{Hg}$ pressure and He pressure on the outside was achieved by making a small hole in the upper part of the Teflon cap.

White X-ray was generated by operating a commercial $\mathrm{W}$ tube at $50 \mathrm{kV}$ and $25 \mathrm{~mA}$. The diffracted X-ray photons from expanded liquid $\mathrm{Hg}$ were detected by a pure-Ge solid-state-detector (SSD) located at $2 \theta$ of $5,7.5,11,15,20,25$ and $35^{\circ}$. Since the spectrum in the energy region $16-42 \mathrm{keV}$ was used for the data analysis, the magnitude of scattering vector, $\mathrm{k}, \mathrm{k}=(4 \pi \sin \theta / \mathrm{hc}) \mathrm{E}$, E:photon energy, h:Planck constant, c:velocity of light, ranged from 0.7 to $13 \mathrm{~A}^{-1}$. A photon accumulating time at scattering angles of $5,7.5,11,15$ and $20^{\circ}$ was one hour and that at 25 and $35^{\circ}$ was two hours.

In order to obtain the structure factor, $\mathrm{S}(\mathrm{k})$, of expanded liquid $\mathrm{Hg}$ from the experimental scattering intensity, several data corrections had to be made such as taking account of the escapeeffect of the Ge SSD, the energy spectrum of the primary X-ray beam, the energy dependence of the polarization of the primary beam, the absorption by the sapphire cell, compressed $\mathrm{He}$ gas, $\mathrm{Be}$ windows and liquid $\mathrm{Hg}$ itself, and finally Compton scattering from liquid $\mathrm{Hg}$ and the sapphire

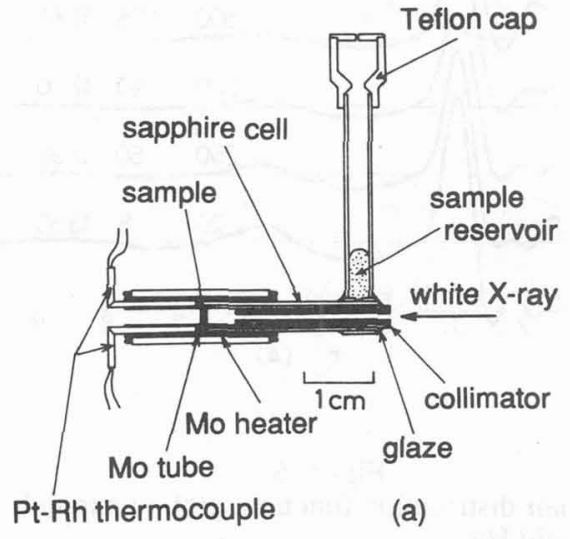

(a)

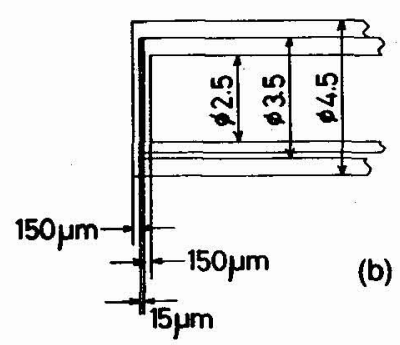

Figure 3.

(a) The construction of the sapphire cell used for $\mathrm{X}$-ray diffraction studies of expanded liquid $\mathrm{Hg}$. (b) Details of the sample space. See text for further details. 
cell $/ 14,16 /$. The first and second corrections are rather unique for the energy-dispersive $\mathrm{X}$-ray diffraction. Detailed descriptions about the procedure of the data analysis are given in the literature $/ 15 /$.

Figure 4 shows $\mathrm{S}(\mathrm{k})$ of liquid $\mathrm{Hg}$ at $20^{\circ} \mathrm{C}$ and 5 bar obtained for the different angle settings. Circles show the $S(k)$ data obtained for angle settings of $2 \theta=5$ and $20^{\circ}$, triangles for $2 \theta=7.5$ and $25^{\circ}$, squares for $2 \theta=11$ and $35^{\circ}$ and reversed-triangles for $2 \theta=15^{\circ}$. As seen in the figure, the consistency of the data in the same k-region is quite good.

\section{Results and discussion}

Figure 5 shows $\mathrm{S}(\mathrm{k})$ of expanded liquid $\mathrm{Hg}$ in the temperature and pressure ranges up to $1450^{\circ} \mathrm{C}$ and

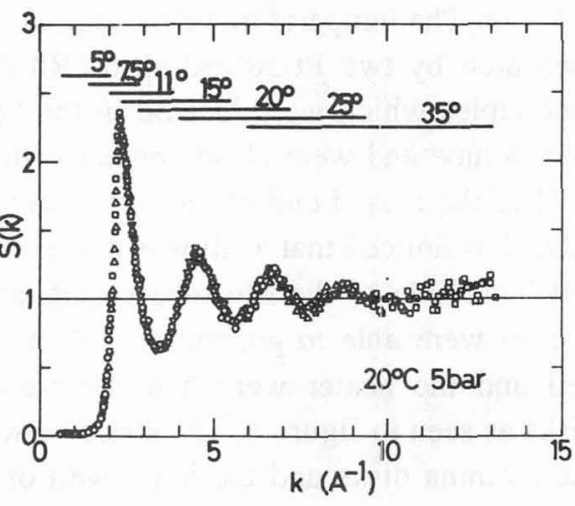

Figure 4.

The structure factor, $S(k)$, of liquid $\mathrm{Hg}$ at $20^{\circ} \mathrm{C}$ and 5 bar obtained for different angle settings. 1750 bar along the saturated vapour-pressure curve and with densities ranging from 13.55 to 8.78 $\mathrm{g} / \mathrm{cm}^{3}$. Note that these results include the data in the M-NM transition region. Dots represent the experimental data and the full curves show the Fourier transforms of the corresponding pair dis-

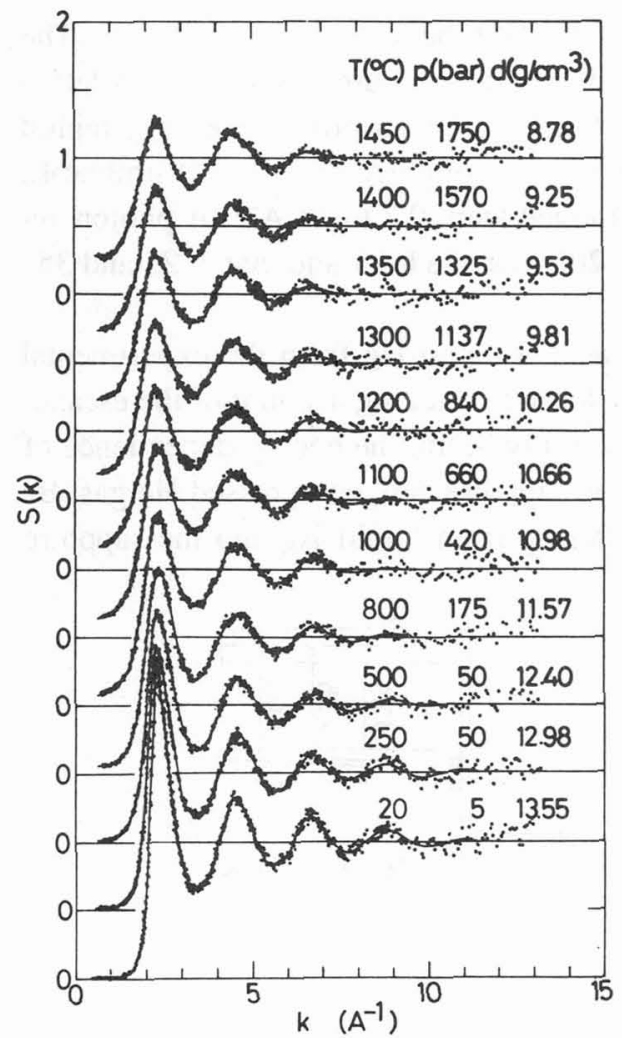

Figure 5.

The structure factor, $S(k)$, of expanded liquid $\mathrm{Hg}$.

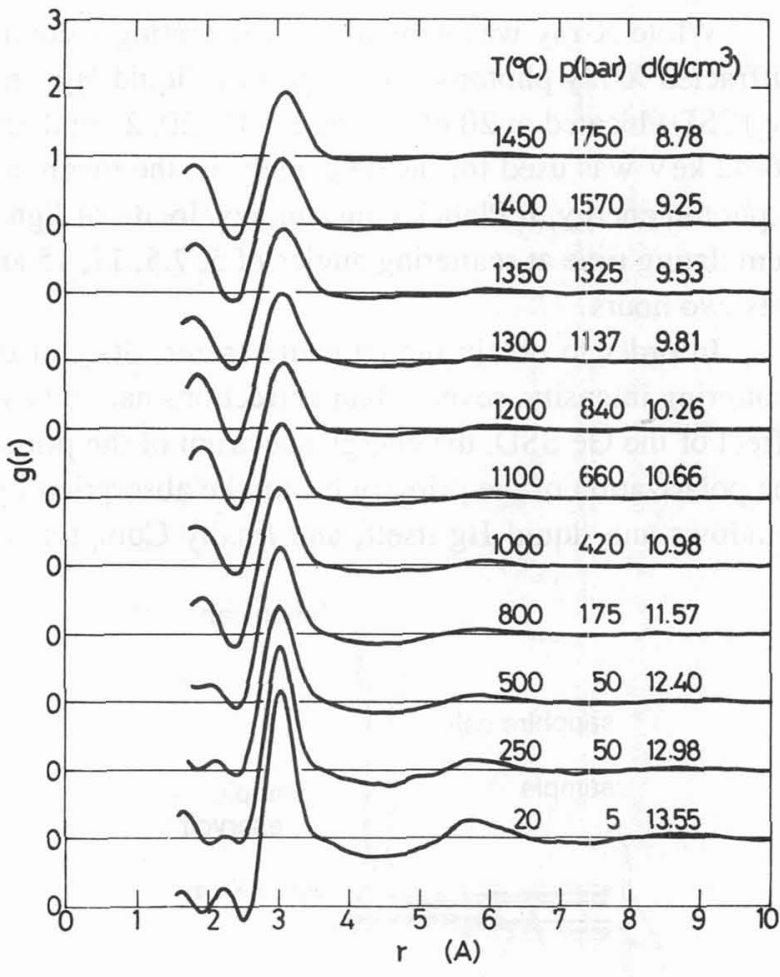

Figure 6.

The pair distribution function, $g(r)$, of expanded liquid Hg. 
tribution functions, $g(r)$, which are shown in figure 6. The $\mathrm{S}(\mathrm{k})$ at $20^{\circ} \mathrm{C}$ and $5 \mathrm{bar}$ is in good agreement with previous data obtained by the usual angle-dispersive method $/ 17 /$. The characteristic feature of the temperature and pressure variations of $S(k)$ is that the oscillation damps and the peak width becomes broad with increasing temperature and pressure. This tendency is noticed in the first peak, whereas the second peak seems to damp rather slowly. As clearly seen in the figure, the values of $S(k)$ at low $k$ region, say at $0.7 \mathrm{~A}^{-1}$, gradually increase with increasing temperature and pressure. Further, there appears the minimum of $S(k)$ in the low $k$ region below $1 \mathrm{~A}^{-1}$ for the data at $1450^{\circ} \mathrm{C}$ and 1750 bar.

Figure 6 shows $g(r)$ deduced from the Fourier transformation of $\mathrm{S}(\mathrm{k})$. In the Fourier transformation, the values of $S(k)$ in the small $k$ region betweeen 0 and $0.7 \mathrm{~A}^{-1}$ were estimated by interporating the values of $S(0)$ and $S(k)$ above $0.7 \mathrm{~A}^{-1}$, where $S(0)$ were calculated using the experimentally obtained isothermal compressibility of expanded liquid $\mathrm{Hg} / 1 /$. With increasing temperature and pressure, the long-range oscillation of $g(r)$ diminishes. The broadening of the first peak gradually occurs but no remarkable change of the peak position is observed. The small peaks of $\mathrm{g}(\mathrm{r})$ in the region $\mathrm{r}<2.5 \mathrm{~A}$ may be due to termination effects on the Fourier transformation.

Figure 7 shows the coordination number, $\mathrm{N}_{1}$, together with the nearest-neighbour distance, $\mathrm{r}_{1}$, as a function of density. As is well known, it is not easy to obtain the definite value of $\mathrm{N}_{1}$ from the diffuse $\mathbf{g}(\mathbf{r})$ pattern of liquid. Because there is overlap between the first- and secondneighbour peak, $\mathrm{N}_{1}$ depends quite sensitively on the method employed to define and integrate the first-neighbour peak. However, variations of $\mathrm{N}_{1}$ as a function of temperature, pressure or density can be determined with more reliability as long as a consistent definition of $\mathrm{N}_{1}$ is used. We employed three different methods as shown at the upper of the figure. The first, (a), is the method integrating the radial distribution function, $4 \pi \mathrm{r}^{2} \rho_{0 \mathrm{~g}}(\mathrm{r})$, up to the maximum position, $r_{\max }$, and taking twice the integral, where $\rho_{0}$ denotes the average number density of $\mathrm{Hg}$. The second, (b), is the method integrating $4 \pi r^{2} \rho_{0 g}(r)$ up to the maximum position of $g(r), r 1$, and taking twice the integral. The third, (c), is a special method taking account the asymmetry of the firstneighbour peak. We regard the triangle area below the tangent line as that due to the penetration of the second-neighbours.

As is clearly seen in figure $7, \mathrm{~N}_{1}$ decreases substantially and almost linearly with decreasing density in the metallic region, that is, down to about $10 \mathrm{~g} / \mathrm{cm}^{3}$, whatever method might be employed. On the contrary, $\mathrm{r}_{1}$ remains constant. This is consistent with our previous results /14/. 
When the M-NM transition is approached, however, the deviation from the linear plots of $\mathrm{N}_{1}$ becomes evident and $\mathrm{r}_{1}$ starts to elongate. From these findings we can speculate how the structural change of liquid $\mathrm{Hg}$ occurs in the microscopic sense with volume expansion. As schematically illustrated in figure 8 , the atomic arrangement indicated by (a) represents a uniform expansion in which $\mathrm{r}_{1}$ increases and $\mathrm{N}_{1}$ remains unchanged. On the contrary, (b) shows an inhomogeneous expansion in which $\mathrm{r}_{1}$ remains constant and $\mathrm{N}_{1}$ decreases. The volume expansion of liquid $\mathrm{Hg}$ in the metallic region occurs in such a way as shown in (b). This is the same as that observed in liquid alkali metals $/ 18,19 /$. However, it is changed to the way as shown in (a) when the M-NM transition region is approached. It is interesting to note that the M-NM transition seems to take place when $\mathrm{N}_{1}$ decreases to a minimum value and $r_{1}$ may be forced to

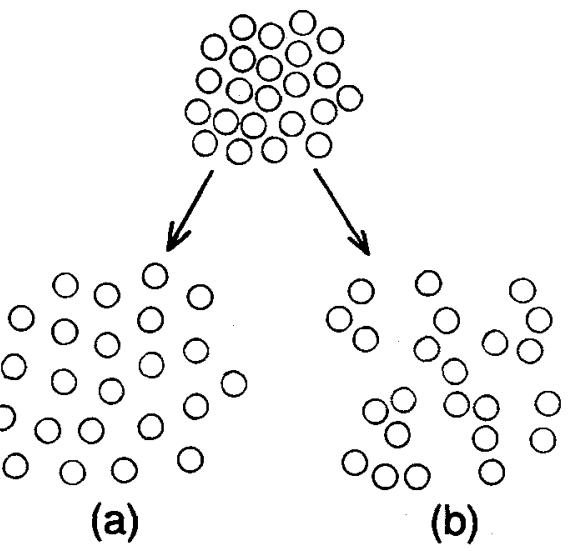

Figure 8.

Schematic drawings of two ways how the structural change of liquid $\mathrm{Hg}$ occurs in the microscopic sense with volume expansion. See text for further details. elongate.

The X-ray diffraction measurements extending to the lower density region, especially under the supercritical condition, are now in progress.

\section{Acknowledgements}

The authors are grateful to Professors H. Endo, M. Watabe and K. Hoshino for valuable discussions. This work is partly supported by the Grant-in-Aid for Scientific Research Fund from the Ministry of Education, Science and Culture of Japan and Toray Science Foundation.

\section{References}

/1/GÖTZLAFF W, SCHÖNHERR G and HENSEL F, Z. Phys. Chem. NF 156 (1988) 219, GÖTZLAFF W, PhD Thesis, University of Marburg 1988

/2/ HENSEL F and FRANK E U, Ber. Bunsenges. Phys. Chem. 70 (1966) 1154

13/ SCHMUTZLER R W and HENSEL F, Ber. Bunsenges. Phys. Chem. 76 (1972) 531

/4/ SCHÖNHERR G, SCHMUTZLER R W and HENSEL F, Philos. Mag. B 40 (1979) 411

/5/ YAO M and ENDO H, J. Phys. Soc. Jpn. 51 (1982) 966

/6/ DUCKERS L J and ROSS R G, Phys. Lett. A 38 (1972) 291

77/ NEALE F E and CUSACK N E, J. Phys. F 9 (1979) 85

/8/ YAO M and ENDO H, J. Phys. Soc. Jpn. 51 (1982) 1504

19/ EVEN U and JORTNER J, Philos. Mag. 25 (1972) 715; Phys. Rev. B 8 (1973) 2536

/10/ IKEZI H, SCHWARZENEGGER K, SIMONS A L, PASSNER A L and MCCALL S L, Phys. Rev. B 18 (1978) 2494

/11/ HEFNER W, SCHMUTZLER R W and HENSEL F, J. Phys. Paris 41 (1980) C8-62

/12/EL-HANANY U and WARREN W W Jr, Phys. Rev. Lett. 34 (1975) 1276

/13/ SUZUKI K, INUTAKE M, FUJIWAKA S, YAO M and ENDO H, J. Phys. Paris 41 (1980) C8-66

/14/ HOSOKAWA S, MATSUOKA T and TAMURA K, J. Phys.: Condens. Matter 3 (1991) in press

/15/ TAMURA K, J. Non-cryst. Solids $117 / 118$ (1990) 450

/16/ NISHIKAWA K and IIJIMA T, Bull. Chem. Soc. Jpn 57 (1984) 1750

/17/ BOSIO L, CORTES R and SEGAUD C, J. Chem. Phys. 71 (1979) 3595

/18/ FRANZ G, FREYLAND W, GLÄSER W, HENSEL F and SCHNEIDER E, J. Phys. Paris 41 (1980) C8-194; FRANZ G, PhD Thesis, University of Marburg 1980

/19/ WINTER R, HENSEL F, BODENSTEINER T and GLÄSER W, Ber. Bunsenges. Phys. Chem. 91 (1987) 